Measurements of the ionisation rates of lithium-like ions

This content has been downloaded from IOPscience. Please scroll down to see the full text. 1977 J. Phys. B: At. Mol. Phys. 103637

(http://iopscience.iop.org/0022-3700/10/18/020)

View the table of contents for this issue, or go to the journal homepage for more

Download details:

IP Address: 130.237.29.138

This content was downloaded on 08/12/2015 at $21: 57$

Please note that terms and conditions apply. 


\title{
Measurements of the ionisation rates of lithium-like ions $\dagger$
}

\author{
E Källneł and L A Jones \\ Los Alamos Scientific Laboratory, University of California, Los Alamos, New Mexico \\ 87545, USA
}

Received 29 March 1977, in final form 11 July 1977

\begin{abstract}
The ionisation rates of lithium-like nitrogen $(\mathrm{N} v)$ and oxygen $(\mathrm{O}$ VI) were measured using a $60 \mathrm{~kJ}$ theta pinch as a plasma light source $\left(T_{\mathrm{c}}=80-150 \mathrm{eV}\right.$, $N_{\mathrm{e}} \sim 1-5 \times 10^{16} \mathrm{~cm}^{-3}$ ). The time evolutions of strong VUV lines were observed with a $2.2 \mathrm{~m}$ grazing-incidence monochromator. Two different plasmas were used $\left(2.5 \mathrm{mT} \mathrm{N} \mathrm{N}_{2}\right.$ and $20 \mathrm{mT}\left(\mathrm{He}+1 \% \mathrm{O}_{2}\right)$ ) and diagnosed with $90^{\circ}$ Thomson scattering $\left(T_{\mathrm{c}}\right)$ and holographic interferometry $\left(N_{\mathrm{c}}\right)$. The plasma was modelled using the coupled rate equations with the semiempirical formula of Kunze for the ionisation rates. The measured ionisation rates for the lithium-like jons were found to be consistent with those calculated by Kunze's semiempirical formula to within $10 \%$. Corrections were made in our model for end losses, temperature dependence of the excitation rates, radial electron density and temperature distributions, lowering of the ionisation limit due to collisions, and overlap of the $2 \mathrm{~s}$ and $2 \mathrm{p}$ levels in the ground-state population.
\end{abstract}

\section{Introduction}

Radiation losses from impurity ions in hot, dense plasmas are limiting factors in controlled fusion research. Light elements like carbon, nitrogen, and oxygen are present as common impurities in large fusion devices as well as heavy elements like molybdenum, iron, gold, etc, which emanate from the limiter and the walls of the plasma devices. All the impurities are highly ionised in the hot plasmas and the degree of ionisation is determined by the ionisation rates and the plasma temperature and density. The radiation losses are determined by the ionisation stages which are most abundant in the plasma. In order to be able to evaluate these losses, it is important to measure quantitatively the rates for the processes responsible for the ionisation stage build-up. Cross sections for collisional ionisation and excitation of lower ionisation stages can be measured by crossed-beam experiments (Dunn 1976). This technique has recently been applied to lithium-like carbon and nitrogen ions (Crandall et al 1977). Another technique which can be used to measure the collisional ionisation and excitation rates is to observe the radiation from ions embedded in a well diagnosed plasma. This has the advantage of an experimental situation closely resembling present-day fusion-plasma devices. On the other hand it is only possible to measure rates, i.e. the product of the cross section and the electron velocity averaged over the Maxwellian electron velocity distribution.

\footnotetext{
$\uparrow$ Work performed under the auspices of the United States Energy Research and Development Administration.

$¥$ Present address: Physics Department, University of British Columbia, Vancouver, BC, Canada.
} 
The method employed in earlier investigations, as well as in the present study, is to use a diagnosed plasma created by a theta pinch as the source of ions and to observe the emitted radiation from the ions. The theta pinch is a suitable plasma device as the pinching of the plasma creates a very fast increase in temperature and density. This sudden increase is much more rapid than the ionisation and excitation processes and consequently the ionisation stage build-up is determined mainly by the collisional ionisation between the electrons and ions. After the first increase in temperature and density there is a slow change in the temperature during the time of interest. The time scale of the plasma, a few microseconds, makes it feasible to diagnose the plasma carefully regarding the temporal and radial dependence of temperature and density. Thus, the modelling of the plasma is relatively easy and by using theoretical or semiempirical input rates for the ionisation, excitation, and recombination processes, it is possible to predict the light output from the plasma. As the temperature might vary as a function of time it is possible to measure the rates over an extended temperature region.

Earlier ionisation rate measurements have recently been reviewed by several authors (Kunze 1972, Burgess et al 1977, Gabriel and Jordan 1972, Kunze and Johnston 1971, Haddad and McWhirter 1973). Lithium-like ions have been studied by Kunze (1971) and by Datla et al (1976). We have previously studied neon including the lithium-like Ne viII (Jones et al 1977). The present paper is an extension of this work covering carbon, nitrogen and oxygen in the attainable ionisation stages. Several ionisation stages of carbon were also observed, i.e. C IV, V and VI, but the time development of the emission from these ions was found to be dominated by the excitation rates and thus closely followed the temperature variations of our plasma. We therefore present these data only to show that our temperature measurements and their incorporation into the model yield reasonable predictions for the time development of the emission from these ions.

\section{Experimental}

\subsection{Theta pinch}

The theta pinch, the capacitor bank and the experimental set-up were the same as those used in our previous work (Jones et al 1977). In the present investigation we found it convenient to use pure nitrogen as the fill gas $(2.5 \mathrm{mT})$ as this produced a plasma with impurity radiation from carbon and oxygen as well. Thus we could diagnose one plasma and study three elements from it. To obtain a higher temperature and also to overlap with the measurements from the nitrogen plasma we studied an oxygen-seeded helium plasma $\left(20 \mathrm{mT}\left(\mathrm{He}+1 \% \mathrm{O}_{2}\right)\right)$. The gas was continuously flowing to compensate for the leakage through the slit of the vUV monochromator in order to maintain a constant pressure in the discharge tube.

\subsection{Diagnostics}

The electron density was measured with holographic interferometry (Jahoda and Siemon 1972). Figures 1 and 2 show the measured electron density as a function of time averaged over the area. It can be seen from the figures that the electron density was very similar for the two plasmas; however, the stability and reproducibility were better for the oxygen-seeded helium plasma than for the nitrogen plasma. 


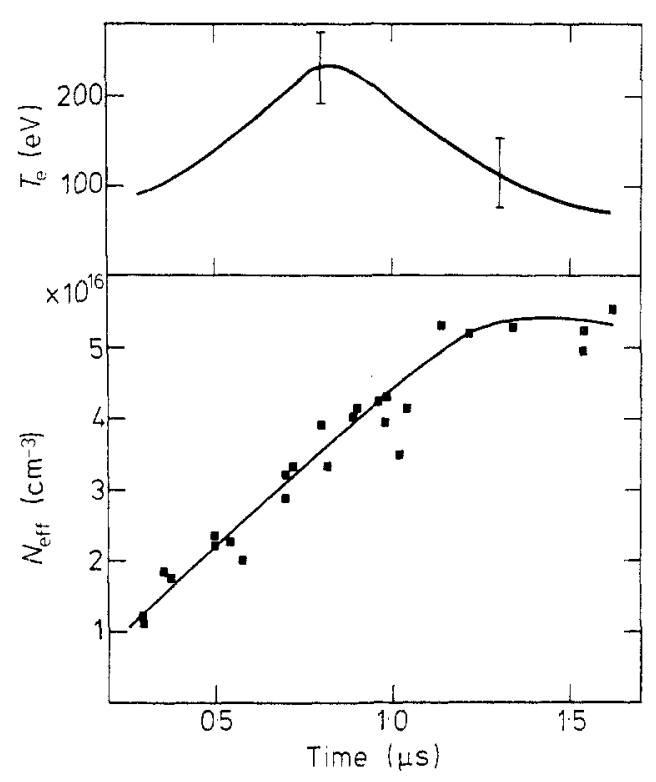

Figure 1. Electron temperature $T_{\mathrm{c}}(\mathrm{eV})$ and electron density $N_{\mathrm{c}}\left(\mathrm{cm}^{-3}\right)$ as a function of time ( $\left.\mu \mathrm{s}\right)$ for the $2.5 \mathrm{mT} \mathrm{N}_{2}$ plasma.

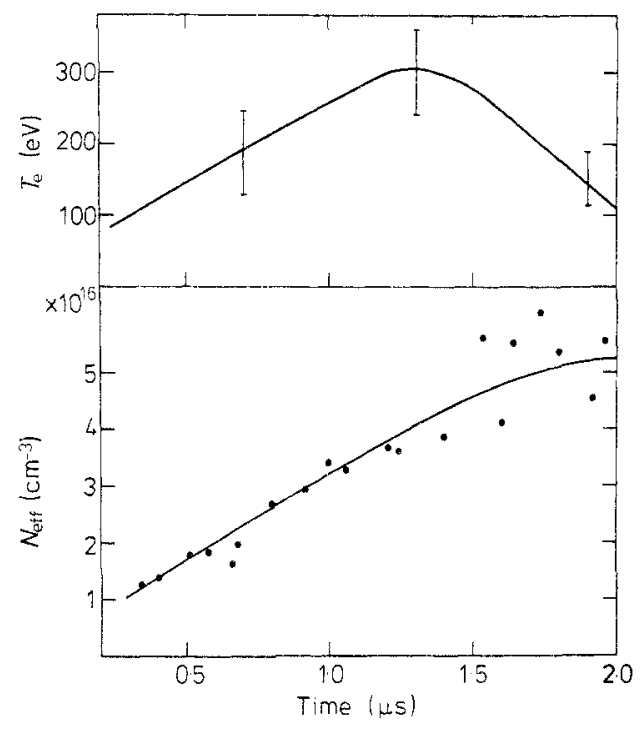

Figure 2. Electron temperature $T_{8}(\mathrm{eV})$ and density $N_{\mathrm{c}}\left(\mathrm{cm}^{-3}\right)$ as a function of time ( $\left.\mu \mathrm{s}\right)$ for the $20 \mathrm{mT}$ $\left(\mathrm{He}+1 \% \mathrm{O}_{2}\right)$ plasma. The error bars represent the maximum scattering of experimental points.

The holograms showed that the position of the plasma centrum varied somewhat from shot to shot for the nitrogen plasma, but the viewing solid angle was made large enough to enable viewing of the total pinched-plasma diameter under all circumstances. The density profiles as a function of radius were plotted at times ranging from $0.3 \mu$ s to $1.3 \mu \mathrm{s}$. The diameter of the compressed nitrogen plasma was about $3.5 \mathrm{~mm}$ and that of the seeded helium plasma was roughly $3 \mathrm{~mm}$.

The electron temperature was measured by means of $90^{\circ}$ Thomson scattering using a $2 \mathrm{~J}$ ruby laser with a $20 \mathrm{~ns}$ pulse width. The profile was scanned on a shot-toshot basis using a $20 \mathrm{~cm}$ monochromator equipped with a holographic grating and a quartz window photomultiplier tube. The focal spot of the laser was about $1 \mathrm{~mm}$ in diameter and was moved along the radius of the plasma to obtain the radial dependence of the electron temperature. Five different radial positions were measured. Figures 1 and 2 show the time dependences of the temperatures at the centre of the tube for the two plasmas. The profiles obtained in the Thomson scattering measurements were fitted by least squares to a Gaussian after having been corrected for the variation in sensitivity of the optical system as calibrated by a tungsten lamp. On each radius the time was scanned in steps of $0.1 \mu$ s from 0.3 to $1.6 \mu$ s after the initiation of the bank discharge. This gave 12-15 data points on each radius and wavelength. Smooth curves were drawn through these points. The radial dependence of the temperature is shown in figure 3. The errors are estimated from the scattering of the experimental points and from the possible uncertainties in the fitting procedure. We estimate the actual error in the temperature to be $\pm 20 \%$. This is smaller than the standard deviation of the temperature measured at one spatial position at one time since we assumed the temperature varied smoothly in time and space, which decreased the uncertainty in our statistics. The holograms were also analysed at the same positions as used in the Thomson scattering measurements. 


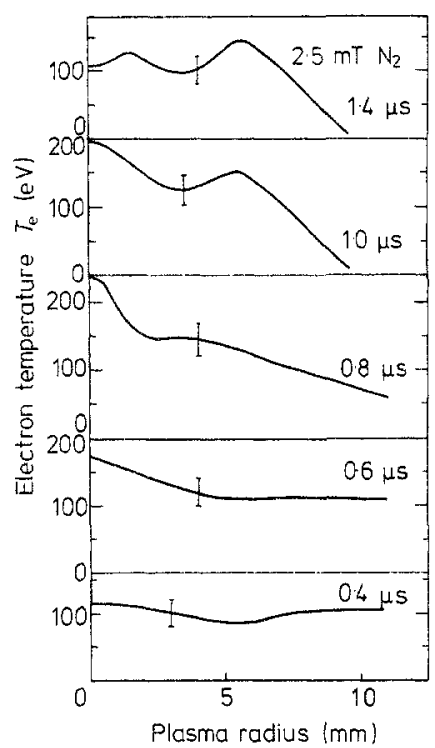

Figure 3. Electron temperature as a function of plasma radius (mm) for different times.

The total intensity of the Thomson scattered light showed the same temporal and radial dependence as did the density obtained from the holograms.

\subsection{VUV emission measurements}

A $2.2 \mathrm{~m}$ grazing-incidence spectrograph was connected to the discharge tube so as to view the plasma radially. The open port was large enough to view the entire plasma except prior to the first compression. The spectra were recorded photographically in order to identify the lines from carbon, nitrogen and oxygen in all the ion stages occurring. The identification was ensured by using methane (carbon), nitrogen and oxygen respectively as fill gases. As already mentioned, we found that the nitrogen plasma had impurities from both carbon and oxygen that made it possible to observe emission from all three elements from the same plasma.

Table 1. Observed vuv emission lines for the studied ion stages.

\begin{tabular}{|c|c|c|c|}
\hline Ion & Configuration $^{a}$ & Wavelength $(\AA)^{a}$ & Transition $^{a}$ \\
\hline $\begin{array}{c}\text { N IV } \\
\mathrm{V} \\
\mathrm{VI}\end{array}$ & $\begin{array}{l}1 s^{2} 2 s^{2}\left({ }^{1} S_{0}\right) \\
1 s^{2} 2 s\left({ }^{2} S_{1 / 2}\right) \\
1 s^{2}\left({ }^{1} S_{0}\right)\end{array}$ & $\begin{array}{l}283.5 \\
209 \cdot 3 \text { (first, second order) } \\
173.9\end{array}$ & $\begin{array}{l}2 \mathrm{~s} 2 \mathrm{p}-2 \mathrm{~s} 3 \mathrm{~d}\left({ }^{3} \mathrm{P}^{\mathrm{o}-}-{ }^{3} \mathrm{D}\right) \\
2 \mathrm{~s}-3 \mathrm{p}\left(g^{2} \mathrm{~S}-{ }^{2} \mathrm{P}^{\circ}\right) \\
1 \mathrm{~s} 2 \mathrm{p}-1 \mathrm{~s} 3 \mathrm{~d}\left({ }^{3} \mathrm{P}^{\mathrm{o}}-{ }^{3} \mathrm{D}\right)\end{array}$ \\
\hline $\begin{array}{c}\mathrm{OIV} \\
\mathrm{V} \\
\mathrm{VI}\end{array}$ & $\begin{array}{l}1 \mathrm{~s}^{2} 2 \mathrm{~s}^{2} 2 \mathrm{p}\left({ }^{2} \mathrm{P}_{1 / 2}^{0}\right) \\
1 \mathrm{~s}^{2} 2 \mathrm{~s}^{2}\left({ }^{1} \mathrm{~S}_{0}\right) \\
1 \mathrm{~s}^{2} 2 \mathrm{~s}\left({ }^{2} \mathrm{~S}_{1 / 2}\right) \\
1 \mathrm{~s}^{2}\left({ }^{1} \mathrm{~S}_{0}\right)\end{array}$ & $\begin{array}{l}238.4 \\
192.8 \text { (first, second order) } \\
173.0 \text { (first, second order) } \\
150.1 \text { (first, second order) } \\
120.3\end{array}$ & $\begin{array}{l}2 \mathrm{p}-3 \mathrm{~d}\left(g^{2} \mathrm{P}^{0}-{ }^{2} \mathrm{D}\right) \\
2 \mathrm{~s} 2 \mathrm{p}-2 \mathrm{~s} 3 \mathrm{~d}\left({ }^{3} \mathrm{P}^{\mathrm{o}}-{ }^{3} \mathrm{D}\right) \\
2 \mathrm{p}-3 \mathrm{~d}\left({ }^{2} \mathrm{P}^{\mathrm{o}}-{ }^{2} \mathrm{D}\right) \\
2 \mathrm{~s}-3 \mathrm{p}\left(g^{2} \mathrm{~S}-{ }^{2} \mathrm{P}^{0}\right) \\
1 \mathrm{~s} 2 \mathrm{~s}-1 \mathrm{~s} 3 \mathrm{p}\left({ }^{3} \mathrm{~S}-{ }^{3} \mathrm{P}^{\mathrm{o}}\right)\end{array}$ \\
\hline $\begin{array}{l}\text { C IV } \\
V \\
V I\end{array}$ & $\begin{array}{l}1 s^{2} 2 s\left({ }^{2} S_{1 / 2}\right) \\
1 s^{2}\left({ }^{1} S_{0}\right) \\
1 s\left({ }^{2} S_{1 / 2}\right)\end{array}$ & $\begin{array}{l}312 \cdot 4 \\
248 \cdot 7 \\
182 \cdot 2\end{array}$ & $\begin{array}{l}2 \mathrm{~s}-3 \mathrm{p}\left(g^{2} \mathrm{~S}-{ }^{2} \mathrm{P}^{o}\right) \\
1 \mathrm{~s} 2 \mathrm{p}-1 \mathrm{~s} 3 \mathrm{~d}\left({ }^{3} \mathrm{P}^{\mathrm{o}}-{ }^{3} \mathrm{D}\right) \\
2-3\end{array}$ \\
\hline
\end{tabular}

a Kelly and Palumbo (1973). 
After the photographic recording, the ion stages to be studied were selected and the strong lines from each ion stage were recorded photoelectrically. Each line was recorded in one shot, using a monochromator resolution of $0.35 \AA$. The reproducibility of the VUV emission made it possible to superimpose three shots on each picture. The lines studied are given in table 1 with wavelengths and assignments from Kelly and Palumbo (1973).

\section{Theoretical}

\subsection{Modelling of the plasma and the light emission}

As in our previous work (Jones et al 1977) we solved the set of coupled rate equations including the relevant corrections for spatial and temporal temperature and density dependences occurring in our plasma. The equation for the change in the number density of the $z$ th ion, $N_{z}$, is

$$
\begin{aligned}
\frac{\mathrm{d} N_{z}(r, t)}{\mathrm{d} t}= & N_{\mathrm{e}}(r, t) N_{z-1}(r, t) S_{z-1}(r, t) \\
& -N_{\mathrm{e}}(r, t) N_{z}(r, t)\left[S_{z}(r, t)+\alpha_{z-1}(r, t)+\delta_{z-1}(r, t)+\gamma_{z-1}(r, t) N_{\mathrm{e}}(r, t)\right] \\
& +N_{\mathrm{e}}(r, t) N_{z+1}(r, t)\left[\alpha_{z}(r, t)+\delta_{z}(r, t)+N_{\mathrm{e}}(r, t) \gamma_{z}(r, t)\right]
\end{aligned}
$$

where $N_{c}(r, t)$ is the measured electron density and $S_{z}(r, t)$ is the ionisation rate from Kunze's semiempirical formula (Kunze 1971, Lotz 1967):

$$
S_{z}(r, t)=7.5 \times 10^{-8} \frac{n_{z}}{E_{z}}\left[\left(\ln \frac{40 k T}{E_{z}}\right)^{3}+40\right] \frac{(k T)^{1 / 2}}{E_{z}+3 k T} \mathrm{e}^{-E_{z} / k T} .
$$

Here $E_{z}$ is the ionisation energy $(\mathrm{eV}), n_{z}$ the number of equivalent electrons, and $k T$ the electron temperature $(\mathrm{eV}) ; \alpha_{z}(r, t)$ is the rate coefficient for radiative recombination taken from Merts et al (1976):

$$
\alpha_{z}(r, t)=7.05 \times 10^{-15} \frac{1}{g_{z}} \sum_{n=n_{0}} \frac{g_{n}}{n} \frac{\left(E_{n}\right)^{2}}{(k T)^{3 / 2}} \mathrm{e}^{E_{n} / k T} E_{1}\left(\frac{E_{n}}{k T}\right)
$$

where $g_{z}$ and $g_{n}$ are the statistical weights of the ground state of the recombining ion and the excited state $n$ of the recombined ion respectively, $E_{n}$ is the binding energy of state $n(\mathrm{eV})$ and $k T$ is the electron temperature $(\mathrm{eV}) ; \delta_{z}(r, t)$ is the rate coefficient for dielectronic recombination and $\gamma_{z}(r, t)$ is the rate coefficient for threebody recombination.

As in our previous work, we integrate over the volume of the plasma viewed assuming that the ion radial density distribution is the same as that of the electrons. Since the axial and theta integrals are constants in time we obtain for the relative rate of change of the total ion number density:

$$
\begin{aligned}
& \frac{\mathrm{d} N_{z}^{T}(t)}{\mathrm{d} t}=\frac{N_{z-1}^{T}(t) \int N_{\mathrm{e}}^{2}(r, t) S_{z-1}(r, t) \mathrm{d} r}{\int N_{\mathrm{e}}(r, t) \mathrm{d} r}-\frac{N_{z}^{T}(t) \int N_{\mathrm{e}}^{2}(r, t)\left[S_{z}(r, t)+\alpha_{z-1}(r, t)\right] \mathrm{d} r}{\int N_{\mathrm{e}}(r, t) \mathrm{d} r} \\
& +\frac{N_{z+1}^{T}(t) \int N_{\mathrm{e}}^{2}(r, t) \alpha_{z}(r, t) \mathrm{d} r}{\int N_{\mathrm{e}}(r, t) \mathrm{d} r}
\end{aligned}
$$


where the three-body and dielectronic recombination rates have been neglected. This assumption is justified since, following Merts et al (1976), the three-body recombination rate for these ions at our plasma conditions is two orders of magnitude smaller than the radiative recombination rate. At the densities prevailing in our plasma, the contribution from the dielectronic recombination is also small. This can be understood since the effect of the collisional limit, i.e. the principal quantum number above which the rate of collisional ionisation is larger than the rate of radiative decay, is to decrease the effective ionisation potential. The contribution to the dielectronic recombination rate will thus decrease since the states above the collisional limit should not be included in the summation for the dielectronic rate (Burgess 1965). Thus the radiative recombination is the only correction required for our conditions and is important only for the high ion stages at late times. Assuming that every excitation leads to the emission of a photon (coronal equilibrium) we get for the light emission,

$$
I(t)=N_{z}^{T}(t) \frac{\int N_{\mathrm{e}}^{2}(r, t) \chi(r, t) \mathrm{d} r}{\int N_{\mathrm{e}}(r, t) \mathrm{d} r}
$$

where $\chi(r, t)$ is the excitation rate coefficient. The temperature dependence of the excitation rate was then taken into account according to Van Regemorter (1962) and McWhirter (1975) by using the formula

$$
\chi(r, t) \propto \frac{1}{(k T)^{1 / 2}} \mathrm{e}^{-E_{r / k} /} .
$$

The end losses were taken into account by letting the number of electrons from the ionised atoms follow the total measured electron density, i.e.

$$
\int N_{\mathrm{e}}(r, t) \mathrm{d} r=\sum_{z}(z-1) N_{z}^{T}(t)
$$

for the nitrogen ions in the nitrogen plasma. For the oxygen and carbon impurities in the nitrogen plasma we used the nitrogen end losses obtained above as input in the plasma modelling program. We compute the necessary integrals including the measured spatial and temporal dependences of the temperature and density. These integrals are then used when solving the rate equations according to equations (4) and (5). The procedure for fitting to the observed light emission is to choose initial conditions and multiply the ionisation rates of Kunze with a factor until the best agreement is found between the observed and predicted profiles.

We also considered effects of optical depths for the observed vUV resonance lines of nitrogen. For $\mathrm{N} \mathrm{v}$, for example, the optical depth is about 3 for our plasma conditions (Griem 1964). However, the optical depth in the axial direction is considerably larger. Therefore most of the light observed in the radial direction will be light originally emitted in the axial direction which finally exits radially after being absorbed and scattered (Julienne and Davis 1973). This tends to steepen the rise and the fall of the vUv light profile but will not change the position of the peak. The oxygen lines in the nitrogen or helium plasma are all optically thin.

The depression of the effective ionisation potential due to the collisional limit was considered by calculating the principal quantum number state above which the rate of collisional ionisation is larger than the rate of radiative decay. For the Li-like ions we also considered the population of the $2 \mathrm{p}$ state by taking an average of the $2 \mathrm{~s}$ and $2 \mathrm{p}$ energies assuming a statistical population. This is appropriate, since 
for the $2 \mathrm{p}-2 \mathrm{~s}$ transition the excitation rate exceeds the radiative rate by about a factor of four (Merts et al 1976, Wiese et al 1966) and the 2p and 2 s levels will then be nearly in collisional equilibrium. In the radiative recombination integrals we included all the states up to the collisional limit. For the temperature correction of the excitation rate we used the corrected excitation energies (considering the $2 \mathrm{p}-2 \mathrm{~s}$ statistical population).

For the helium-like ions the metastable triplet state has to be considered also. Following Datla et al (1976), we calculated the effect of the metastable state of N VI to be negligible for our plasma conditions $\left(200 \mathrm{eV}, 2 \times 10^{16} \mathrm{~cm}^{-3}\right)$.

\section{Results and discussion}

The results for the fits are given in figures $4-7$. The time scale starts at the pinch of the plasma, $0.3 \mu$ s after the initiation of the main bank current, since the earlier time developments of the emitted light profiles are totally determined by plasma dynamics. The experimental light profiles were fitted mainly to the rise, the peak, and the first fall. This is appropriate as the late time profiles are dominated by long-lasting tails that might be emitted from a 'halo' of the plasma and are not predicted by the present model. Also part of the tail can be associated with a background emission that has not been subtracted from the raw data. We have seen effects of long-lasting tails also for krypton (Jones and Källne 1977) and earlier thetapinch experiments have seen this effect as well (Thomas et al 1972). The positions of the peaks are sensitive to the ionisation rates for the lithium-like ions while for the highest ion stages ( $\mathrm{CV}, \mathrm{VI}, \mathrm{N}$ VI, O vi) the predicted light profiles were found to be insensitive to the ionisation rates. The determining factor in the light profile for these ion stages is the excitation rate, or rather the temperature dependence of the excitation rate. This can be understood when one considers that the actual electron temperature at the relevant times (figures 1 and 2) is comparable to the ionisation potential of these ions. This means that the rate of population of the excited states is the dominant factor in the light emission profile. The emission profiles for the carbon ions were found to be insensitive within experimental error to a change in

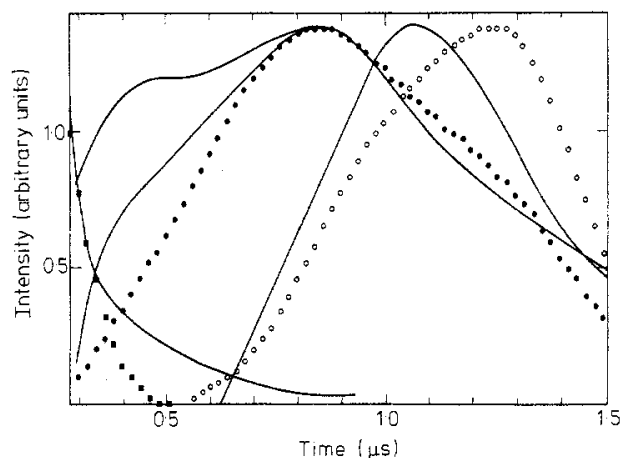

Figure 4. Experimental (-) and theoretical $(\boldsymbol{a}, 0,0)$ vUV light profiles for $\mathrm{C}$ IV, $\mathrm{v}$, and VI for the $2.5 \mathrm{mT} \mathrm{N}_{2}$ plasma. The intensities are normalised at peak value. $A$ N IV profile has been subtracted from the $\mathrm{C} v$ profile as described in the text.

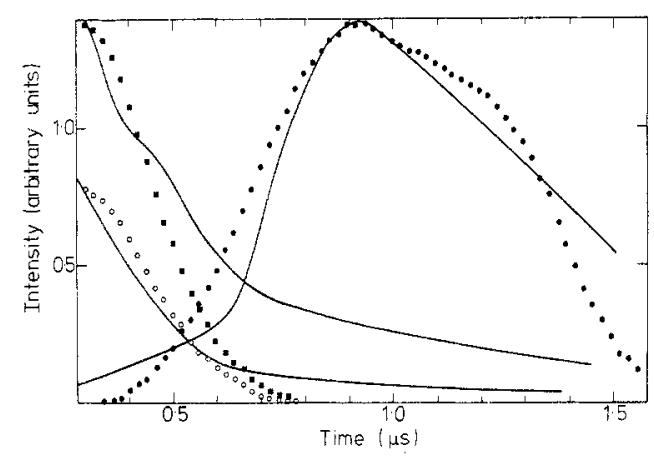

Figure 5. Experimental $(-)$ and theoretical $(0, \square$, light emission profiles for $\mathrm{N} \mathrm{IV}, \mathrm{V}$, and VI for the $2.5 \mathrm{mT} \mathrm{N}_{2}$ plasma as a function of time. The intensities are normalised at peak value. 


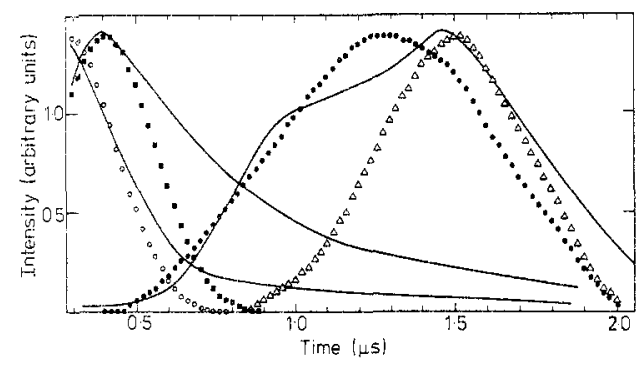

Figure 6. Experimental (--) and theoretical $(\boldsymbol{\bullet}, \triangle, \mathbf{\square})$ light emission profiles for $\mathrm{O} \mathrm{IV}, \mathrm{v}$, and v1 for the $2.5 \mathrm{mT} \mathrm{N}_{2}$ plasma. The intensities are normalised at peak value.

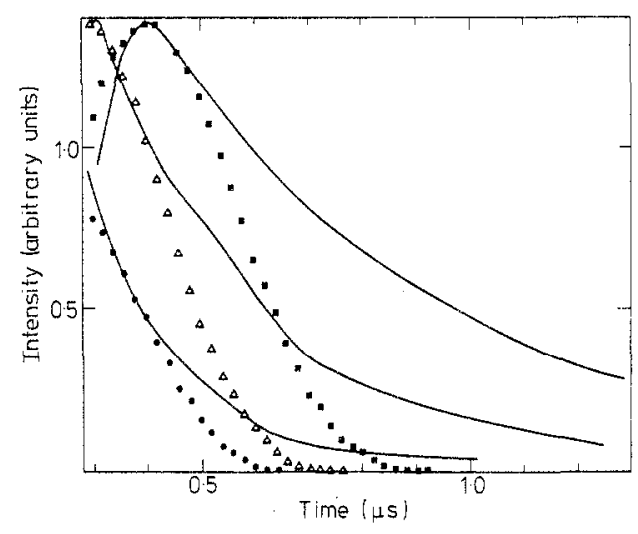

Figure 7. Experimental (-) and theoretical $(O, \boldsymbol{m}, \Delta)$ vUv light profiles for $\mathrm{O} v, \mathrm{VI}, \mathrm{VII}$, and vill (only theoretical) for the $20 \mathrm{mT}\left(\mathrm{He}+1 \% \mathrm{O}_{2}\right.$ ) plasma. The intensities are normalised at peak value.

the ionisation rates by at least a factor of two. Thus the fits achieved for carbon demonstrate the accuracy of the temperature measurements where the exponential temperature dependence in the excitation rate (equation (6)) is the dominating factor. The time development of the emission from $\mathrm{CV}(248.7 \AA)$ has a contribution from N IV $(248.6 \AA)$. We have thus subtracted a reasonable intensity of the N IV time profile in order to get the $\mathrm{C} v$ profile.

The multiplicative factors and the initial conditions which were used in the fits are presented in table 2. The initial conditions were chosen so that the predicted relative light outputs of the first two ion stages at the very early times $(0.3 \mu \mathrm{s})$ were in agreement with experiment. The main error in the fit comes from the choice of the initial conditions. For each variation of the multiplicative factor for the ionisation rate we changed the initial conditions so as to arrive at the best fit. This sensitivity to initial conditions implies that the errors in our rate measurements are

Table 2. The initial number densities and the multiplicative factors for the ionisation rates used in the fitting program.

\begin{tabular}{|c|c|c|c|c|}
\hline & \multicolumn{2}{|c|}{ Multiplicative factors } & \multicolumn{2}{|c|}{ Initial population } \\
\hline & $2.5 \mathrm{mT} \mathrm{N}_{2}$ & $20 \mathrm{mT}\left(\mathrm{He}+1 \% \mathrm{O}_{2}\right)$ & $2.5 \mathrm{mT} \mathrm{N}_{2}$ & $20 \mathrm{mT}\left(\mathrm{He}+1 \% \mathrm{O}_{2}\right)$ \\
\hline $\mathrm{N}$ III & 0.1 & & 0 & \\
\hline IV & 0.9 & & $4 \times 10^{15}$ & \\
\hline $\mathrm{v}$ & 1.5 & & $3 \times 10^{15}$ & \\
\hline $\mathrm{VI}_{\mathrm{I}}$ & 1 & & 0 & \\
\hline O III & 1 & 1 & 0 & 0 \\
\hline IV & $0 \cdot 15$ & 0.1 & $8 \times 10^{12}$ & 0 \\
\hline v & 0.45 & 0.35 & $5.5 \times 10^{13}$ & $3 \times 10^{15}$ \\
\hline $\mathrm{VI}$ & 0.9 & 0.9 & $8.7 \times 10^{13}$ & $4 \times 10^{15}$ \\
\hline VII & & 1.5 & 0 & 0 \\
\hline vIII & & 1 & & \\
\hline C. III & 0.4 & & 0 & \\
\hline IV & 0.7 & & $3 \times 10^{13}$ & \\
\hline $\mathrm{v}$ & 1.5 & & $4 \times 10^{13}$ & \\
\hline VI & 1 & & 0 & \\
\hline
\end{tabular}


larger than for our earlier work for neon. The multiplicative factor for the lithium-like ions was found to be $0.9 \pm 0.3$ where the error includes the largest acceptable variation in the fitting procedure combined with a $20 \%$ error in the temperature and density. It is interesting to compare the rates obtained for the lithium-like ions with earlier measurements (Kunze 1971, Datla et al 1976) and with the calculations of Diichs and Griem (1966). With the corrections discussed earlier our measured rates are in agreement with Kunze's semiempirical formula but in disagreement with his earlier measured values that were obtained at higher temperatures. Assuming the temperature dependence suggested by Seaton (1962), we get an ionisation rate coefficient $z_{z}$ in analogy with Düchs and Griem (1966) as shown in table 3. E is the ionisation energy of $\mathrm{O}$ vI decreased due to the collisional limit as mentioned previously. In the last column, corrections have been made to the depressed ionisation potential as a result of $2 \mathrm{~s}-2 \mathrm{p}$ ground-state population mixing. It is clear that the semiclassical dipole approximation gives rates that are too low by about a factor of three.

Table 3. Ionisation rate coefficients $z_{z}$ for $O$ vi. $S_{z}=o_{z}(T / E)(1+T / E)^{-1} \exp (-E / T)$. where $E$ is the ionisation energy $(\mathrm{eV}), T$ the electron temperature $(\mathrm{eV})$, and $S_{z}$ the ionisation rate $\left(\mathrm{cm}^{3} \mathrm{~s}^{-1}\right)$.

\begin{tabular}{llll}
\hline & & \multicolumn{2}{c}{ Present } \\
\cline { 3 - 4 } & $\begin{array}{l}\text { Düchs and } \\
\text { Griem (1966) }\end{array}$ & $\begin{array}{l}\text { Lowering of } \\
\text { ionisation potential }\end{array}$ & $\begin{array}{l}\text { 2s-2p ground- } \\
\text { state mixing }\end{array}$ \\
\hline$E(\mathrm{eV})$ & 128 & 128 & 119 \\
$\mathrm{~s}_{2}\left(\mathrm{~cm}^{3} \mathrm{~s}^{-1}\right)$ & $6.1 \times 10^{-10}$ & $22.0 \times 10^{-10}$ & $25.8 \times 10^{-10}$ \\
\hline
\end{tabular}

In table 4 we summarise the ionisation rates for the lithium-like ions as given by the semiempirical formula (Kunze 1971), by earlier experiments (Kunze 1971, Datla et al 1976, Jones et al 1977), and by the present investigation. In the first row of the table are given the ionisation potentials for the studied lithium-like ions (C IV, $\mathrm{N} \mathrm{v}, \mathrm{O}$ VI, and $\mathrm{Ne}$ VIII). The ratios between the electron temperature and the ionisation potential for the different experiments are given in the rows just preceding the experimental rate results. Earlier experiments have revealed ionisation rate measurements for $\mathrm{C}$ Iv, $\mathrm{Nv}$, and $\mathrm{O}$ vi. The present study includes $\mathrm{Nv}$ and $\mathrm{O}$ vi while we have previously reported measurements for Ne vill. The comparison between the semiempirical formula and experimental ionisation rates shows that the rates measured at lower temperatures are in better agreement with the semiempirical formula than those measured at higher temperatures.

There are several experimental differences between the earlier experiments and the present one which might explain the discrepant results. We will discuss the differences and also the two corrections we have applied to the Kunze semiempirical formula to meet the conditions of our plasma. The earlier experiments have been performed at lower densities, which can explain why the corrections important in this experiment are negligible. The conditions for the present ionisation rate measurements are not ideal as the ion stage build-up is fast for the lithium-like ions and the higher ion stages were found to be completely dominated by excitation. The high density is also evident from the magnitude of the correction to the ionisation rate 
Table 4. Ionisation rates for lithium-like ions.

\begin{tabular}{|c|c|c|c|c|c|}
\hline & C.IV & $\mathrm{Nv}$ & \multicolumn{2}{|l|}{$\mathrm{OVI}$} & Ne vill \\
\hline $\begin{array}{l}\text { Ionisation } \\
\text { potential }(\mathrm{eV})\end{array}$ & $64 \cdot 49$ & 97.88 & \multicolumn{2}{|l|}{138} & 239 \\
\hline$k T_{\mathrm{e}} / E_{\mathrm{i}}$ & 2.33 & 2.04 & \multicolumn{2}{|l|}{1.45} & \\
\hline $\begin{array}{l}\text { Experimental rate } \\
\left(\mathrm{cm}^{3} \mathrm{~s}^{-1}\right)\end{array}$ & $\begin{array}{l}1.52 \times 10^{-9} a \\
1.74 \times 10^{-9 b}\end{array}$ & $\begin{array}{l}0.63 \times 10^{-9 a} \\
1.08 \times 10^{-9 b}\end{array}$ & \multicolumn{2}{|c|}{$0.35 \times 10^{-9}$} & \\
\hline $\begin{array}{l}\text { Theoretical rate } \\
\left(\mathrm{cm}^{3} \mathrm{~s}^{-1}\right)\end{array}$ & $\begin{array}{l}2.4 \times 10^{-9} d \\
1.29 \times 10^{-9}\end{array}$ & $\begin{array}{l}1.2 \times 10^{-9 c} \\
0.67 \times 10^{-9 d}\end{array}$ & \multicolumn{2}{|c|}{$\begin{array}{l}0.59 \times 10^{-9} a \\
0.31 \times 10^{-9 d}\end{array}$} & \\
\hline$k T_{\mathrm{e}} / E_{\mathrm{i}}$ & & 1.02 & 0.79 & 0.88 & 0.95 \\
\hline $\begin{array}{l}\text { Experimental rate } \\
\left(\mathrm{cm}^{3} \mathrm{~s}^{-1}\right)\end{array}$ & & $\begin{array}{l}9.70 \times 10^{-10} \\
5.8 \times 10^{-10 b}\end{array}$ & $3.91 \times 10^{-10}$ & $4.35 \times 10^{-10}$ & $1 \cdot 10 \times 10^{-10 y}$ \\
\hline $\begin{array}{l}\text { Theoretical rate } \\
\left(\mathrm{cm}^{3} \mathrm{~s}^{-1}\right)\end{array}$ & & $\begin{array}{r}6.59 \times 10^{-10 \mathrm{c}} \\
8.59 \times 10^{-10 \mathrm{e}} \\
10.78 \times 10^{-10 \mathrm{f}}\end{array}$ & $\begin{array}{l}2.82 \times 10^{-10 \mathrm{c}} \\
3.53 \times 10^{-10 \mathrm{e}} \\
4.34 \times 10^{-10 \mathrm{f}}\end{array}$ & $\begin{array}{l}3.20 \times 10^{-10 c} \\
3.96 \times 10^{-10 c} \\
4.84 \times 10^{-10}\end{array}$ & $1.58 \times 10^{-10}$ \\
\hline
\end{tabular}

"Kunze (1971).

${ }^{b}$ Crandall et al (1977).

- According to Kunze (1971) and Lotz (1967).

${ }^{d}$ According to Burgess (1964).

' Kunze's semiempirical formula (Kunze 1971) corrected for depression of ionisation potential ( $\mathrm{N} \vee 88.49 \mathrm{eV}$ and $\mathrm{O}$ vi $128 \cdot 0 \mathrm{eV}$ ).

${ }^{\mathrm{f}}$ Kunze's semiempirical formula (Kunze 1971) corrected for depression of ionisation potential and $2 \mathrm{~s}-2 \mathrm{p}$ ground-state population ( $\mathrm{N}$ v $80.94 \mathrm{eV})$ and $(\mathrm{O}$ vi $119.03 \mathrm{eV})$.

Jones et al (1977).

formula. First, the depression of the ionisation limit decreases the ionisation potential by about $10 \%$ which leads to a $25 \%$ increase in the ionisation rates. We have estimated that the contribution from excitation of the ground state to levels above the collision limit ( $n=6$ for these lithium-like ions) is of the same order of magnitude as the increase in the ionisation rate due to the decreased ionisation potential. Second, the inclusion of the $2 \mathrm{p}$ level to the ground-state population also increases the ionisation rate due to the effective decrease of the ionisation potential. These two corrections are the important factors for the ionisation rate measurements for our conditions. Stepwise excitation followed by ionisation from the $3 \mathrm{~d}$ levels is negligible since the radiative decay is fast enough to prevent any appreciable population of the $3 \mathrm{~d}$ level. The atomic structure of the lithium-like ions is simple, since there are no metastable states to take into consideration. Furthermore, any double-ionisation process is negligible for these few-electron systems. Thus the present results give $0.9 \pm 0.3$ as a correction to the ionisation rates predicted by the Kunze semiempirical formula. Our earlier ionisation rate measurements on lithium-like neon were more sensitive to the change in ionisation rates and thus gave a more accurate value for the multiplicative factor. Recently, beam experiments have been reported for lithium-like carbon and nitrogen (Crandall et al 1977). The measured ionisation cross sections can be compared with the rate measurements after integrating the cross sections over a Maxwellian electron distribution. These computed values are also included in table 
4 for comparison. We can thus see from the table that the ion-beam experiments give rates that fall somewhere between earlier experiments and the calculated rates of Lotz (1967). On the other hand the present plasma experiment with a temperature closer to the threshold energy tends to give higher ionisation rates. This could be further evidence that the ionisation rate formulae have the wrong temperature dependence as discussed recently by Datla et al (1976).

\section{Conclusion}

The ionisation rates for lithium-like nitrogen and oxygen have been measured and found to agree with Kunze's semiempirical formula to within $10 \%$ when the formula is corrected for the depression of the ionisation limit and the collisional population of the $2 \mathrm{~s}$ and $2 \mathrm{p}$ levels. These corrections to the ionisation rate formula are found to be important for the present plasma conditions. Earlier plasma measurements as well as ion-beam measurements on lithium-like carbon and nitrogen tend to give a larger correction to the semiempirical formula when the rates are measured at higher temperatures. Thus the temperature dependence in the ionisation rate formula seems to be inconsistent with the present experimental data around threshold and with the earlier data at higher temperatures.

\section{Acknowledgments}

We wish to thank $\mathrm{D}$ Thomson for fruitful discussions and encouragement during the course of the work. We also appreciate the technical assistance of R Roy.

\section{References}

Burgess A 1964 Proc. Symp. Atomic Collision Processes in Plasmas, Culham; AERE Report 4818 p 63

-1965 Proc. 2nd Harvard-Smithsonian Conf'. on Stellar Aimospheres ed O Gingerich p 47

Burgess A H, Summers H P, Cochrane D M and McWhirter R W P 1977 Mon. Not. R. Astron. Soc. 179 275-92

Crandall D H, Taylor P O and Phaneuf R A 1977 Proc. 10th Int. Conf. on Physics of Electronic and Atomic Collisions, Paris, July 1077 Abstracts to be published

Datla R U, Nugent L J and Griem H R 1976 Phys. Rev. A 14 979-83

Düchs D and Griem H R 1966 Phys. Fluids 9 1099-109

Dunn G H 1976 IEEE Trans. Nucl. Sci. NS-23 pp 929--33

Gabriel A H and Jordan C 1972 Case Studies in Atomic Collision Physics vol 2 pp 211-91

Griem H R 1964 Plasma Spectroscopy (New York: McGraw-Hill) chap 6

Haddad G N and McWhirter R W P 1973 J. Phys. B: Atom. Molec. Phys, 6 715-27

Jahoda F C and Siemon R E 1972 Los Alamos Scientific Laboratory Informal Report LA-5058-MS

Jones L A and Källne E 1977 J. Phys. B: Atom. Molec. Phys. to be submitted

Jones L A, Källne E and Thomson D B 1977 J. Phys. B: Atom. Molec. Phys. $10187-95$

Julienne P S and Davis J 1973 Naval Research Laboratory Report NRL-2556

Kelly R L and Palumbo L J 1973 Naval Research Laboratory Report NRL-7599

Kunze H J 1971 Phys. Rev. A 3 937-72

1972 Space Sci. Rev. $13565-83$

Kunze H J and Johnston W D 1971 Phys. Rev. A 3 1384-93

Lotz W 1967 Astrophys. J. Suppl. 14 207-38

McWhirter R W P 1975 Course on Plasma Diagnostics and Data Acquisition Systems ed A Eubank and E Sindoni 
Merts A L, Cowan R D and Magee N H Jr 1976 Los Alamos Scientific Laboratory Informal Report LA-6220-MS

Seaton M J 1962 Atomic and Molecular Processes ed D R Bates (New York: Academic Press) chap 11

Thomas K S, Harder C R, Quinn W E and Siemon R E 1972 Phys. Fluids 15 1658-66

Van Regemorter H 1962 Astrophys. J. 136 906-15

Wiese W L, Smith M W and Glennon G M 1966 Atomic Transition Probabilities NBS Report No NSRDS-NBS-4, vol 1 (Washington DC: NBS) 